\title{
molecules
}

ISSN 1420-3049

http://www.mdpi.org

Full Paper

\section{Synthesis and Analgesic Activity Evaluation of Some Agmatine Derivatives}

\author{
Hongxia He ${ }^{1}$, Mengjia Liu ${ }^{1}$, Zhibing Zheng ${ }^{2, *}$, Ying Liu ${ }^{2}$, Junhai Xiao ${ }^{2}$, Ruibin Su ${ }^{2}$, Chun \\ $\mathrm{Hu}^{1}$, Jin $\mathrm{Li}^{2}$ and Song $\mathrm{Li}^{2}$ \\ ${ }^{1}$ School of Pharmaceutical Engineering, Shenyang Pharmaceutical University, Shenyang, 110016, \\ P.R. China \\ 2 Department of Medicinal Chemistry, Beijing Institute of Pharmacology and Toxicology, Beijing, \\ 100850, P.R. China
}

E-mail addresses: Zhibing ZHENG: zbzheng@nic.bmi.ac.cn, Mengjia Liu: mj32800@yahoo.com.cn,

Ying Liu: luxin314@sina.com, Junhai Xiao: xiaojunhai@yahoo.com.cn, Ruibin Su:

Ruibinsu@Yahoo.com, Chun HU: Chem2000@163.com, Jin Li: lijin@nic.bmi.ac.cn, Song LI: lis@nic.bmi.ac.cn

*Author to whom correspondence should be addressed; e-mail: hhx110015@yahoo.com.cn

Received: 10 May 2006 / Accepted: 9 June 2006 / Published: 12 June 2006

\begin{abstract}
A series of $N, N^{\prime}$-disubstituted-2-nitroethene-1,1-diamine and $N, N$ '-disubstituted$N$ ',-cyanoguanidine derivatives were prepared and evaluated for in vivo analgesic activity. The blood brain barrier (BBB) VolSurf model was used to predict the BBB permeation profiles of our synthesized compounds. Some compounds show both remarkable analgesic activity and good BBB permeation profiles, and these compounds might be developed for treatment of opioid tolerance and dependence.
\end{abstract}

Keywords: Agmatine derivatives; synthesis; analgesic activity; blood-brain barrier; opioid dependence.

\section{Introduction}

Agmatine (1, Figure 1) is an endogenous ligand of imidazoline receptors [1], and is biologically active in the nervous system and many other tissues in mammals [2]. Previous research indicates that this compound, which is not able to interact with opioid receptors, plays an important role in regulating the pharmacological actions of opioids [3-6]. Agmatine has weak analgesic effects and shows biphasic modulation on opioid functions, which enhance opioid analgesia, but inhibit tolerance 
to and substance dependence on opioids. The mechanisms associated with the analgesic effect and biphasic modulation on opioid functions is by activation of imidazoline receptors on central nervous system [7-8]. These results suggest that agmatine might be developed for treatment of opioid tolerance and dependence, but the pharmacokinetic profile of agmatine, such as its low ability to cross the blood-brain barrier and its fast excretion (mostly due to its hydrophilicity), restricts its potential drug use [9-10].

\section{Figure 1.}<smiles>N=C(N)NCCCCN</smiles>

\section{1}

It is known that lipophilicity is an essential feature for the penetration of a molecule through the blood-brain barrier [11], consequently, the purpose of the present work was to synthesize several structurally-related agmatine derivatives with better lipophilicity and biological activity. We synthesized some derivatives of agmatine by substituting guanidine with 2-nitroethene-1,1-diamine or $\mathrm{N}$-cyanoguanidine, and tested their analgesic activity in mice in order to find new potent compounds for treatment of opioid tolerance and dependence.

\section{Results and Discussion}

\section{Chemistry}

We used 1,1-bis(methylthio)-2-nitroethene as starting material, which was refluxed with one equivalent of amine to give the corresponding N-substituted-1-(methylthio)-2-nitroethenamines 2a-b. Then compounds 2 were reacted with the appropriate amine to obtain the corresponding N,N'disubstituted -2-nitroethene-1,1-diamine derivatives 3a-j (Scheme 1) [12-13].

Scheme 1. The synthesis of N,N'-disubstituted -2-nitroethene-1,1-diamines.

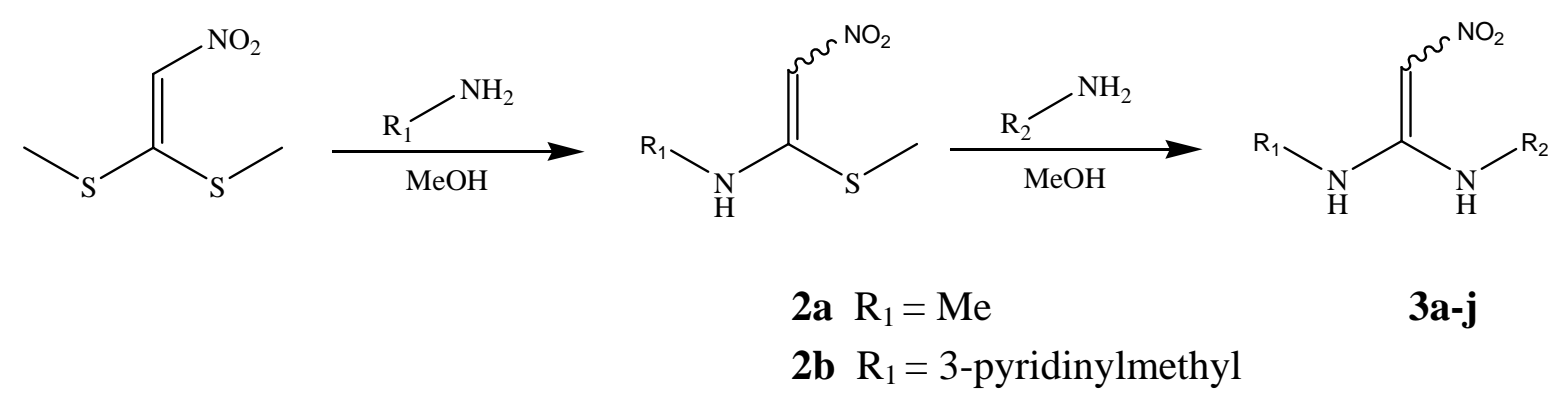

The preparation of N,N'-disubstituted-N' '-cyanoguanidine derivatives 5a-l was similar to that of 3a-j. First, dimethyl N-cyanodithioiminocarbonate was refluxed with one equivalent of amine to give the corresponding methyl N'-cyano-N-substituted-imidothiocarbamates 4a-d. Then compounds 4a-d were reacted with the second amine to give the derivatives 5a-l (Scheme 2) [14-15]. The structures of the compounds are presented in Table 1. 
Scheme 2. The synthesis of N,N'-disubstituted-N' '-cyanoguanidines.<smiles>CSC(=NC#N)SC</smiles><smiles>[R][C@H](C)N</smiles><smiles>CNC(=NN)SC</smiles><smiles>[R]NCCC(C)N</smiles><smiles>[R]NC(=NN)N[R]</smiles>

$$
\begin{array}{ll}
\text { 4a } & \mathrm{R}_{1}=\text { methyl } \\
\text { 4b } & \mathrm{R}_{1}=3 \text {-pyridinylmethyl } \\
\text { 4c } & \mathrm{R}_{1}=\text { isobutyl } \\
\text { 4d } & \mathrm{R}_{1}=\text { phenylethyl }
\end{array}
$$

\begin{tabular}{|c|c|c|c|c|c|}
\hline Compd. & $\mathbf{R}_{\mathbf{1}}$ & $\mathbf{R}_{2}$ & Compd. & $\mathbf{R}_{\mathbf{1}}$ & $\mathbf{R}_{2}$ \\
\hline 3a & $\mathrm{CH}_{3}$ & $\mathrm{CH}_{2} \mathrm{C}\left(\mathrm{CH}_{3}\right)_{2} \mathrm{CH}_{2} \mathrm{~N}\left(\mathrm{CH}_{3}\right)_{2}$ & $5 \mathbf{b}$ & $\mathrm{CH}_{3}$ & $\left(\mathrm{CH}_{2}\right)_{4} \mathrm{NH}_{2}$ \\
\hline $3 \mathbf{b}$ & $\mathrm{CH}_{3}$ & $\left(\mathrm{CH}_{2}\right)_{4} \mathrm{NH}_{2}$ & $5 c$ & $\mathrm{CH}_{3}$ & $\left(\mathrm{CH}_{2}\right)_{5} \mathrm{NH}_{2}$ \\
\hline $3 c$ & $\mathrm{CH}_{3}$ & $\left(\mathrm{CH}_{2}\right)_{5} \mathrm{NH}_{2}$ & $5 d$ & $\mathrm{CH}_{3}$ & $\left(\mathrm{CH}_{2}\right)_{6} \mathrm{NH}_{2}$ \\
\hline 3d & $\mathrm{CH}_{3}$ & $\left(\mathrm{CH}_{2}\right)_{6} \mathrm{NH}_{2}$ & $5 e$ & $\mathrm{CH}_{3}$ & $\left(\mathrm{CH}_{2}\right)_{7} \mathrm{NH}_{2}$ \\
\hline $3 \mathbf{e}$ & $\mathrm{CH}_{3}$ & $\left(\mathrm{CH}_{2}\right)_{4} \mathrm{CH}_{3}$ & $5 f$ & 3-pyridinylmethyl & $\mathrm{CH}_{2} \mathrm{C}\left(\mathrm{CH}_{3}\right)_{2} \mathrm{CH}_{2} \mathrm{~N}\left(\mathrm{CH}_{3}\right)_{2}$ \\
\hline $3 f$ & $\mathrm{CH}_{3}$ & 4-piperidinobutyl & $5 g$ & 3-pyridinylmethyl & $\left(\mathrm{CH}_{2}\right)_{6} \mathrm{NH}_{2}$ \\
\hline $3 g$ & $\mathrm{CH}_{3}$ & $\mathrm{CH}_{2} \mathrm{CCCH}_{2} \mathrm{NH}_{2}$ & $5 h$ & 3-pyridinylmethyl & $\left(\mathrm{CH}_{2}\right)_{7} \mathrm{NH}_{2}$ \\
\hline $3 \mathbf{h}$ & 3-pyridinylmethyl & $\mathrm{CH}_{3}$ & $5 \mathbf{i}$ & isobutyl & $\left(\mathrm{CH}_{2}\right)_{6} \mathrm{NH}_{2}$ \\
\hline $3 \mathbf{i}$ & 3-pyridinylmethyl & $\left(\mathrm{CH}_{2}\right)_{4} \mathrm{NH}_{2}$ & $5 \mathbf{j}$ & isobutyl & $\left(\mathrm{CH}_{2}\right)_{7} \mathrm{NH}_{2}$ \\
\hline $3 \mathbf{j}$ & 3-pyridinylmethyl & $\left(\mathrm{CH}_{2}\right)_{5} \mathrm{NH}_{2}$ & $5 k$ & phenylethyl & $\left(\mathrm{CH}_{2}\right)_{5} \mathrm{NH}_{2}$ \\
\hline $5 a$ & $\mathrm{CH}_{3}$ & $\mathrm{CH}_{2} \mathrm{C}\left(\mathrm{CH}_{3}\right)_{2} \mathrm{CH}_{2} \mathrm{~N}\left(\mathrm{CH}_{3}\right)_{2}$ & 51 & phenylethyl & $\left(\mathrm{CH}_{2}\right)_{7} \mathrm{NH}_{2}$ \\
\hline
\end{tabular}$$
\text { 5a-1 }
$$

Table 1. Formulae of compounds 3a-i and $\mathbf{5 a - j}$.

\section{Analgesic activity}

The mechanisms associated with the analgesic effect and biphasic modulation on opioid functions is similar, so the analgesic activity of synthesized derivatives can partly shows its ability for treatment of opioid tolerance and dependence. Analgesic activity was evaluated by measuring the effect of the tested compounds on acetic acid-induced writhing in mice. The results showed that compounds $\mathbf{3 d}$, $\mathbf{3 h}$, $\mathbf{5 b}, \mathbf{5 d}, \mathbf{5 e}, \mathbf{5 f}$ and $\mathbf{5 h}$ have notable analgesic activity (see Table 2).

\section{Prediction of blood-brain barrier permeation of the compounds}

The blood-brain barrier (BBB) is a complex cellular system and its function is to maintain the homeostasis of the central nervous system (CNS) by separating the brain from the systemic blood circulation. For drugs targeting the CNS, BBB penetration is a necessity. The BBB Volsurf model [1618] was used to predict the permeation profile of our synthesized compounds and the results indicate that some are potentially good candidates $(-0.15<\mathrm{BBB}<1.00)$ for effective BBB permeation (Table 2 ). 
The model was able to correctly predict the $\mathrm{BBB}$ range for more than $75 \%$ of the compounds. Bearing in mind that $\mathrm{BBB}$ permeation is not only dependent on passive diffusion but also on active transport and metabolism, the results are very encouraging.

Table 2. Inhibition on acetic acid-induced mice writhing and BBB profile as predicted by Volsurf.

\begin{tabular}{|c|c|c|c|c|c|}
\hline Compd & $\begin{array}{c}\text { inhb.rate } \\
\mathbf{( \% )}\end{array}$ & $\begin{array}{c}\text { BBB prediction } \\
\text { by Volsurf }\end{array}$ & Compd & $\begin{array}{c}\text { inhb.rate } \\
\mathbf{( \% )}\end{array}$ & $\begin{array}{c}\text { BBB prediction } \\
\text { by Volsurf }\end{array}$ \\
\hline Saline & 0 & & $5 a$ & 27.0 & 0.79 \\
Morphine & 100 & & $5 b$ & 57.3 & -0.51 \\
Agmatine & 55.3 & -0.64 & $5 c$ & 19.9 & -0.47 \\
3a & $35.8^{*}$ & 0.95 & $5 d$ & $54.1^{* *}$ & -0.34 \\
3b fumarate & 37.7 & -0.28 & $5 e$ & 53.1 & -0.28 \\
3c fumarate & 46.5 & -0.18 & $5 f$ fumarate & $56.2^{*}$ & 0.08 \\
3d fumarate & $52.0^{*}$ & -0.12 & $5 g$ fumarate & 37.3 & -0.74 \\
3e & $33.6^{*}$ & 0.83 & $5 h$ fumarate & 51.3 & -0.69 \\
3f & 23.9 & 0.82 & 5 fumarate & $38.4^{*}$ & -0.28 \\
3g & 35.4 & -0.35 & $5 j$ fumarate & 24.8 & -0.21 \\
3h & $60.6^{*}$ & 0.64 & $5 \mathrm{k}$ fumarate & 4.14 & -0.38 \\
\hline 3i fumarate & 47.9 & -0.64 & $5 l$ fumarate & $16.1^{*}$ & -0.46 \\
\hline 3j fumarate & 27.8 & -0.58 & & & \\
\hline
\end{tabular}

** $\mathrm{P}<0.01, * \mathrm{P}<0.05$, saline and morphine is the control.

\section{Conclusions}

Ten compounds 3a-j and twelve compounds 5a-l were prepared as shown in Schemes 1 and 2. Compounds 3d, $\mathbf{3 h}$ and $\mathbf{5 f}$ present notable analgesic activity and good blood-brain barrier permeation profiles. Further research on these compounds and their potential use for treatment of opioid tolerance and dependence will be report in due course.

\section{Acknowledgements}

The authors wish to express their gratitude to the National Basic Research Program of China (973 project: 2003CB515400) for financial support of this research.

\section{Experimental}

\section{General}

${ }^{1} \mathrm{H}-\mathrm{NMR}$ (400 MHz) and ${ }^{13} \mathrm{C}-\mathrm{NMR}$ (100 MHz) spectra were recorded on a JNM-ECA-400 spectrometer in the solvents indicated. Mass spectral analyses were carried out using a Micromass 
ZabSpec or an API3000 mass spectrometer. Elemental analysis was carried at the CarloErba-1106 instrument. Melting points were recorded on an Electrothermal digital capillary melting point apparatus and are uncorrected. Column chromatography was performed with silica gel H (250-400 mesh).

General procedure for the preparation of compounds 2a-b

A solution of 1,1-bis(methylthio)-2-nitroethene $(50 \mathrm{mmol})$ in absolute methanol $(200 \mathrm{~mL})$ was refluxed with one equivalent of amine for $8 \mathrm{~h}$. After cooling to room temperature, the solvent was evaporated and the residue was recrystallized from ethanol/diethyl ether (1:6) to yield 2a-b.

N-Methyl-1-(methylthio)-2-nitroethenamine (2a) [19]: Yield 65\%; mp: 113-115².

1-(Methylthio)-2-nitro- $N$-[(pyridin-3-yl)methyl]ethenamine (2b) [20]: Yield 67\%; mp: 130-132 ${ }^{\circ} \mathrm{C}$; EIMS: $225.0(\mathrm{M})^{+}$.

General procedure for the preparation of compounds 3a-j and their fumarates

A mixture of N-substituted-1-(methylthio)-2-nitroethenamine 2 (1 mmol) and $\mathrm{MeOH}$ (15 mL) containing 1.5 equivalents of the appropriate amine was refluxed for $6 \mathrm{~h}$. After cooling to room temperature, the solvent was evaporated and the residue was purified by column chromatography to afford the product 3. One equivalent each of fumaric acid and 3 were dissolved in $\mathrm{EtOH}$ and then refluxed for $2 \mathrm{~min}$. After cooling to room temperature diethyl ether was slowly added to the solution. The precipitate formed was filtered off to give the fumarate of $\mathbf{3}$.

$N^{1}, N^{1}, 2,2-T e t r a m e t h y l-N^{3}$-[1-(methylamino)-2-nitrovinyl]propane-1,3-diamine (3a): Yield 69\%; mp: 99-100 ${ }^{\circ} \mathrm{C} .{ }^{1} \mathrm{H}-\mathrm{NMR}\left(\mathrm{D}_{2} \mathrm{O}\right)$ \%: 0.97 (s, 6H), 2.26 (s, 6H), 2.36 (s, 2H), 2.90 (s, 3H), 3.14 (s, 2H), 6.86 (s, $1 \mathrm{H})$; EI-MS: $231.1(\mathrm{M}+1)^{+}$; Anal. Calcd. for $\mathrm{C}_{10} \mathrm{H}_{22} \mathrm{~N}_{4} \mathrm{O}_{2}$ : C, 52.15; H, 9.63; N, 24.33. Found: C, 51.96; H, 9.73; N, 24.16.

$N^{1}$-[1-(Methylamino)-2-nitrovinyl]butane-1,4-diamine (3b). Yield 56\%; mp: 104-106 ${ }^{\circ} \mathrm{C}$; mp fumarate: 110-112 ${ }^{\circ} \mathrm{C} ;{ }^{1} \mathrm{H}-\mathrm{NMR}\left(\mathrm{D}_{2} \mathrm{O}\right)$ 8: 1.47-1.62 (m, 4H), 2.65 (s, $\left.2 \mathrm{H}\right), 2.90$ (s, 3H), 3.28 (t, 2H), 6.88 (s, 1H); FAB-MS: $189.0(\mathrm{M}+1)^{+}$.

$N^{1}$-[1-(Methylamino)-2-nitrovinyl]pentane-1,5-diamine (3c). Yield $57 \%$; mp: $110-112{ }^{\circ} \mathrm{C} ;{ }^{1} \mathrm{H}-\mathrm{NMR}$ $\left(\mathrm{D}_{2} \mathrm{O}\right)$ 8: 1.35-1.48 (m, 6H), 2.61 (t, 2H), 2.89 (s, 3H), 3.26 (t, 2H), 6.87 (s, 1H); EI-MS: $203.0(\mathrm{M}+1)^{+}$; Anal. Calcd. for fumarate $\mathrm{C}_{8} \mathrm{H}_{18} \mathrm{~N}_{4} \mathrm{O}_{2} \cdot \mathrm{C}_{4} \mathrm{H}_{4} \mathrm{O}_{4}$ : C, 45.28; $\mathrm{H}, 6.97 ; \mathrm{N}, 17.60$. Found: C, 44.94; H, 6.98; N, 17.26.

$N^{1}$-[1-(Methylamino)-2-nitrovinyl]hexane-1,6-diamine (3d) [21]. Yield $55 \%$; mp: 111-113 ${ }^{\circ} \mathrm{C}$; ${ }^{1} \mathrm{H}$ NMR $\left(\mathrm{D}_{2} \mathrm{O}\right) \delta: 1.15-1.45$ (m, 8H), 2.61 (t, 2H), 2.89 (s, 3H), 3.26 (t, 2H), 6.87 (s, 1H); EI-MS: 217.2 
$(\mathrm{M}+1)^{+}$; Anal. Calcd. for fumarate $\mathrm{C}_{9} \mathrm{H}_{20} \mathrm{~N}_{4} \mathrm{O}_{2} \cdot \mathrm{C}_{4} \mathrm{H}_{4} \mathrm{O}_{4}$ : C, 46.98; H, 7.28; N, 16.86. Found: C, 46.73; H, 7.33; N, 16.58 .

$N$-Methyl-2-nitro- $N$ '-pentylethene-1,1-diamine (3e). Yield: $69 \%$; mp: 78-80 ${ }^{\circ} \mathrm{C} ;{ }^{1} \mathrm{H}-\mathrm{NMR}$ (DMSO-D ${ }_{6}$ ) $\delta$ : $0.84(\mathrm{~m}, 4 \mathrm{H}), 1.30(\mathrm{t}, 3 \mathrm{H}), 1.62(\mathrm{~s}, 2 \mathrm{H}), 2.90(\mathrm{~s}, 3 \mathrm{H}), 3.24(\mathrm{t}, 2 \mathrm{H}), 6.88(\mathrm{~s}, 1 \mathrm{H})$; EI-MS: $187.2(\mathrm{M})^{+}$.

N-Methyl-2-nitro-N'-[4-(piperidin-1-yl)butyl]ethene-1,1-diamine (3f). Yield 39\%; mp: 139-140 $\mathrm{C}$; ${ }^{1} \mathrm{H}-\mathrm{NMR}\left(\mathrm{CDCl}_{3}\right)$ \&: 1.57-1.68 (m, 10H), 2.38 (s, 6H), 2.88 (d, 3H), 3.20 (s, 2H), $5.53(\mathrm{~s}, 1 \mathrm{H}), 5.90$ (s, 1H), 6.60 (s, 1H); FAB-MS: $257.2(\mathrm{M}+1)^{+}$.

$N^{1}$-[1-(Methylamino)-2-nitrovinyl]but-2-yne-1,4-diamine (3g). Yield 43\%; mp: $146-147^{\circ} \mathrm{C} ;{ }^{1} \mathrm{H}-\mathrm{NMR}$ $\left(\mathrm{D}_{2} \mathrm{O}\right) \delta$ : 2.96 (s, 3H), 3.41 (s, 2H), 4.13 (s, 2H), 6.97 (s, 1H); FAB-MS: $185.2(\mathrm{M}+1)^{+}$.

N-Methyl-2-nitro-N'-[(pyridin-3-yl)methyl]ethene-1,1-diamine (3h) [22]. Yield: 61\%; mp: 161-163 ${ }^{\circ}$; ${ }^{1} \mathrm{H}-\mathrm{NMR}\left(\mathrm{D}_{2} \mathrm{O}\right)$ 8: 2.94 (s, 3H), 4.52 (s, 2H), 6.73 (s, 1H), 7.38 (dd, 1H), 7.73 (d, 1H), 8.41 (d, 2H); ESI-MS: $209.2(\mathrm{M}+1)^{+}$; Anal. Calcd. for $\mathrm{C}_{9} \mathrm{H}_{12} \mathrm{~N}_{4} \mathrm{O}_{2}$ : C, 51.92; H, 5.81, N 26.91. Found: C, 51.88; $\mathrm{H}$, 5.92; N, 26.67.

$N^{1}$-[1-((Pyridin-3-yl)methylamino)-2-nitrovinyl]butane-1,4-diamine (3i). Yield: 61\%; mp fumarate: 197-198 ${ }^{\circ} \mathrm{C} ;{ }^{1} \mathrm{H}-\mathrm{NMR}\left(\mathrm{D}_{2} \mathrm{O}\right) \delta: 1.53$ (s, 4H), 2.84 (s, 2H), 3.22 (s, 2H), 4.46 (s, 2H), 6.34 (s, 1H), 7.30 (t, $1 \mathrm{H}), 7.65(\mathrm{~d}, 1 \mathrm{H}), 8.32(\mathrm{~s}, 2 \mathrm{H})$; EI-MS: $266.0(\mathrm{M}+1)^{+}$; Anal. Calcd. for fumarate $\mathrm{C}_{12} \mathrm{H}_{19} \mathrm{~N}_{5} \mathrm{O}_{2} \cdot 1 / 2$ $\mathrm{C}_{4} \mathrm{H}_{4} \mathrm{O}_{4}$ : C, 52.00; H, 6.55; N, 21.66. Found: C, 51.89; H, 6.61; N, 21.40.

$N^{1}$-[1-((Pyridin-3-yl)methylamino)-2-nitrovinyl]pentane-1,5-diamine (3j). Yield: $55 \%$; mp fumarate: 182-184 ${ }^{\circ} \mathrm{C} ;{ }^{1} \mathrm{H}-\mathrm{NMR}\left(\mathrm{D}_{2} \mathrm{O}\right) \delta: 1.48$ (s, 6H), 2.79 (s, 2H), 3.19 (s, 2H), 4.47 (s, 2H), 6.34 (s, 1H), 7.31 (t, 1H), $7.66(\mathrm{~d}, 1 \mathrm{H}), 8.33$ (s, 2H). EI-MS: $280.0(\mathrm{M}+1)^{+}$.

\section{General procedure for the preparation of $\mathbf{4 a - d}$}

A solution of dimethyl $\mathrm{N}$-cyanodithioiminocarbonate $(50 \mathrm{mmol})$ in absolute methanol $(200 \mathrm{~mL})$ was refluxed with one equivalent of amine $(50 \mathrm{mmol})$ for $6 \mathrm{~h}$. After cooling to room temperature, the solvent was evaporated and the residue was recrystallized from ethanol/diethyl ether (1:6) to yield 4a-l.

Methyl N'-cyano-N-methyl-imidothiocarbamate (4a) [23]: Yield: 59 \%; mp: 202-203 C.

Methyl N'-cyano- $N$-[(pyridin-3-yl)methyl]-imidothiocarbamate (4b) [24]: Yield: 63\%; mp: 155-157 ${ }^{\circ} \mathrm{C}$; ${ }^{1} \mathrm{H}-\mathrm{NMR}\left(\mathrm{DMSO}_{6} \mathrm{D}_{6}\right.$ ) $\delta: 2.62$ (s, 3H), 4.51 (s, 2H), 7.36 (dd, 1H), 7.70 (d, 1H), 8.48-8.52 (m, 2H), $8.91(\mathrm{~s}, 1 \mathrm{H})$.

Methyl N'-cyano-N-isobutyl-imidothiocarbamate (4c) [25]: Yield: 67 \%; mp: $119-121^{\circ} \mathrm{C}$.

Methyl N'-cyano-N-phenylethyl-imidothiocarbamate (4d): Yield: 61 \%; mp: 173-174 ${ }^{\circ}$ (Lit. [26] 172$\left.175^{\circ} \mathrm{C}\right)$. 
General procedure for the preparation of 5a-l and their fumarates

A mixture of methyl N'-cyano-N-substituted-imidothiocarbamate 4a-d (1 mmol) and $\mathrm{MeOH}$ (15 $\mathrm{mL}$ ) containing 1.5 equivalent of amine was refluxed for $6 \mathrm{~h}$. After cooling to room temperature, the solvent was evaporated and the residue was purified by column chromatography to afford the products 5a-l. One equivalent each of fumaric acid and 5a-l was dissolved in EtOH and then refluxed for 2 min. After cooling to room temperature diethyl ether was added slowly to the solution. The precipitate formed was filtered off to give the corresponding fumarate of 5a-l.

$N$ '-Cyano- $N$-[3-(dimethylamino)-2,2-dimethylpropyl]-N'-methylguanidine (5a): Yield 73\%; mp: 124$126^{\circ} \mathrm{C}$; ${ }^{1} \mathrm{H}-\mathrm{NMR}\left(\mathrm{D}_{2} \mathrm{O}\right) \delta$ : 0.97 (s, 6H), 2.33 (s, 6H), 2.36 (s, 2H), 2.77 (s, 3H), 3.09 (s, 2H); EI-MS: $211.1(\mathrm{M})^{+}$; Anal. Calcd. for $\mathrm{C}_{10} \mathrm{H}_{21} \mathrm{~N}_{5}$ : C, 56.84; H, 10.02; N, 33.14. Found: C, 57.00; H, 10.26; N, 33.13.

$N$-(4-Aminobutyl)- $N$ ',-cyano- $N$ '-methylguanidine (5b) [27]: Yield 57\%; mp: $113-115^{\circ} \mathrm{C}$; ${ }^{1} \mathrm{H}-\mathrm{NMR}$ $\left(\mathrm{D}_{2} \mathrm{O}\right) \delta$ : 1.50-1.58 (m, 4H), $2.67(\mathrm{t}, 2 \mathrm{H}), 2.78(\mathrm{~s}, 3 \mathrm{H}), 3.30(\mathrm{t}, 2 \mathrm{H})$; EI-MS: $169.0(\mathrm{M})^{+}$; Anal. Calcd. for $\mathrm{C}_{7} \mathrm{H}_{15} \mathrm{~N}_{5}$ : C, 49.68; H, 8.93; N, 41.38. Found: C, 49.99; H, 8.99; N, 41.18.

$N$-(5-Aminopentyl)- $N$ ' '-cyano- $N$ '-methylguanidine (5c): Yield 56\%; mp: $112-114{ }^{\circ} \mathrm{C} ;{ }^{1} \mathrm{H}-\mathrm{NMR}\left(\mathrm{D}_{2} \mathrm{O}\right)$ $\delta$ : 1.35-1.37 (m, 2H), 1.50-1.57 (m, 4H), 2.64 (t, 2H), 2.77 (s, 3H), 3.18 (t, 2H); EI-MS: 182.0 (M-1) Anal. Calcd. for $\mathrm{C}_{8} \mathrm{H}_{17} \mathrm{~N}_{5}$ : C, 52.43; H, 9.35; N,38.22. Found: C, 52.66; H, 9.46; N, 38.02.

$N$-(6-Aminohexyl)- $N$ ' '-cyano- $N$ '-methylguanidine (5d): Yield 53\%; mp: 78-80 ${ }^{\circ} \mathrm{C} ;{ }^{1} \mathrm{H}-\mathrm{NMR}\left(\mathrm{D}_{2} \mathrm{O}\right) \delta$ : 1.35 (m, 4H), 1.48-1.55 (m, 4H), 2.62 (t, 2H), 2.78 (s, 3H), 3.18 (t, 2H); EI-MS: $197.0(\mathrm{M})^{+}$.

$N$-(7-Aminoheptyl)- $N$ ' '-cyano- $N$ '-methylguanidine (5e): Yield 49\%; mp: 83-86 ${ }^{\circ} \mathrm{C} ;{ }^{1} \mathrm{H}-\mathrm{NMR}\left(\mathrm{D}_{2} \mathrm{O}\right) \delta$ : 1.17-1.33 (m, 10H), $2.41(\mathrm{t}, 2 \mathrm{H}), 2.58(\mathrm{~s}, 3 \mathrm{H}), 2.98(\mathrm{t}, 2 \mathrm{H})$; EI-MS: $211.0(\mathrm{M})^{+}$; Anal. Calcd. for $\mathrm{C}_{10} \mathrm{H}_{21} \mathrm{~N}_{5}$ : C, 56.84; H, 10.02; N, 33.14. Found: C, 56.69; H, 10.17; N, 32.66.

$N$ ',-Cyano- $N$-[3-(dimethylamino)-2,2-dimethylpropyl]-N'-[(pyridin-3-yl)methyl]guanidine (5f): Yield 68\%; mp fumarate: $119-120^{\circ} \mathrm{C} ;{ }^{1} \mathrm{H}-\mathrm{NMR}\left(\mathrm{D}_{2} \mathrm{O}\right) \delta$ : $0.74(\mathrm{~s}, 6 \mathrm{H}), 2.70-2.76(\mathrm{~m}, 8 \mathrm{H}), 3.00(\mathrm{~s}, 2 \mathrm{H}), 4.43$ (s, 2H), 6.33 (m, 2H), 7.59 (m, 1H), 8.05 (m, 1H), 8.39-8.44 (m, 2H); EI-MS: 288.0(M) ${ }^{+}$;

$N$-(6-Aminohexyl)- $N$ '-cyano- $N$ '-[(pyridin-3-yl)methyl]guanidine (5g): Yield 61\%; mp fumarate: 158$159^{\circ} \mathrm{C} ;{ }^{1} \mathrm{H}-\mathrm{NMR}\left(\mathrm{D}_{2} \mathrm{O}\right) \delta: 1.13(\mathrm{~m}, 4 \mathrm{H}), 1.36-1.43(\mathrm{~m}, 4 \mathrm{H}), 2.75(\mathrm{t}, 2 \mathrm{H}), 3.03(\mathrm{t}, 2 \mathrm{H}), 4.40$ (s, 2H), 6.38 (s, 2H), $7.62(\mathrm{~m}, 1 \mathrm{H}), 8.04(\mathrm{~m}, 1 \mathrm{H}), 8.41(\mathrm{~m}, 2 \mathrm{H})$; EI-MS: $274.2(\mathrm{M})^{+}$; Anal. Calcd. for fumarate $\mathrm{C}_{14} \mathrm{H}_{22} \mathrm{~N}_{6} \cdot \mathrm{C}_{4} \mathrm{H}_{4} \mathrm{O}_{4}$ : C, 55.37; H, 6.71; N, 21.52. Found: C, 55.24; H, 6.85; N, 21.42.

$N$-(7-Aminoheptyl)- $N$ ' '-cyano- $N$ '-[(pyridin-3-yl)methyl] guanidine (5h): Yield 66\%; mp fumarate: 149$150^{\circ} \mathrm{C} ;{ }^{1} \mathrm{H}-\mathrm{NMR}\left(\mathrm{D}_{2} \mathrm{O}\right) \delta$ : $1.11(\mathrm{~m}, 6 \mathrm{H}), 1.32-1.45(\mathrm{~m}, 4 \mathrm{H}), 2.75(\mathrm{t}, 2 \mathrm{H}), 3.02(\mathrm{t}, 2 \mathrm{H}), 4.38(\mathrm{~s}, 2 \mathrm{H})$, $6.36(\mathrm{~s}, 2 \mathrm{H}), 7.55(\mathrm{~m}, 1 \mathrm{H}), 7.96(\mathrm{~m}, 1 \mathrm{H}), 8.38(\mathrm{~m}, 2 \mathrm{H})$; EI-MS: $288.4(\mathrm{M})^{+}$; Anal. Calc. for fumarate $\mathrm{C}_{15} \mathrm{H}_{24} \mathrm{~N}_{6} \cdot \mathrm{C}_{4} \mathrm{H}_{4} \mathrm{O}_{4}$ : C, 56.42; H, 6.98; N, 20.78. Found: C, 56.56; H, 7.20; N, 21.22. 
$N$-(6-Aminohexyl)- $N$ ',-cyano- $N$ '-isobutylguanidine (5i): Yield 57\%; mp fumarate: $154-157^{\circ} \mathrm{C}$; ${ }^{1} \mathrm{H}$ NMR $\left(\mathrm{D}_{2} \mathrm{O}\right) \delta$ : $0.74(\mathrm{~d}, 6 \mathrm{H}), 1.22$ (m, 4H), 1.40-1.50 (m, 4H), 1.52 (m, 1H), 2.84 (dd, 4H), 3.05 (t, 2H), 6.41 (s, 2H); EI-MS: $239.2(\mathrm{M})^{+}$; Anal. Calcd. for fumarate $\mathrm{C}_{12} \mathrm{H}_{25} \mathrm{~N}_{5} \cdot \mathrm{C}_{4} \mathrm{H}_{4} \mathrm{O}_{4}$ : C, 54.07; H, 8.22; N, 19.70. Found: C, 54.60; H, 8.09; N, 19.97.

$N$-(7-Aminoheptyl)- $N$ ',-cyano- $N$ '-isobutylguanidine (5j): Yield $51 \%$; mp fumarate: $113-115^{\circ} \mathrm{C}$; ${ }^{1} \mathrm{H}$ NMR $\left(\mathrm{D}_{2} \mathrm{O}\right) \delta$ : $0.74(\mathrm{~d}, 6 \mathrm{H}), 1.21(\mathrm{~s}, 4 \mathrm{H}), 1.39-1.51(\mathrm{~m}, 4 \mathrm{H}), 1.69(\mathrm{~m}, 1 \mathrm{H}), 2.84(\mathrm{dd}, 4 \mathrm{H}), 3.05(\mathrm{t}, 2 \mathrm{H})$, 6.54 (s, 2H); EI-MS: $253.2(\mathrm{M})^{+}$; Anal. Calcd. for fumarate $\mathrm{C}_{13} \mathrm{H}_{27} \mathrm{~N}_{5} \cdot \mathrm{C}_{4} \mathrm{H}_{4} \mathrm{O}_{4}: \mathrm{C}, 55.27 ; \mathrm{H}, 8.46$; N, 18.96. Found: C, 55.11; H, 8.55; N, 19.14 .

$N$-(5-Aminopentyl)- $N$ ',-cyano- $N$ '-phenethylguanidine (5k): Yield 55\%; mp fumarate: $155-156{ }^{\circ} \mathrm{C}$; ${ }^{1} \mathrm{H}$ NMR $\left(\mathrm{D}_{2} \mathrm{O}\right) \delta$ : 1.09-1.22 (m,4H), $1.42(\mathrm{dd}, 2 \mathrm{H}), 2.67-2.87(\mathrm{~m}, 6 \mathrm{H}), 3.28(\mathrm{t}, 2 \mathrm{H}), 6.49(\mathrm{~s}, 2 \mathrm{H}), 7.09-$ 7.19 (m, 5H); EI-MS: $273.2(\mathrm{M})^{+}$; Anal. Calc. for fumarate $\mathrm{C}_{15} \mathrm{H}_{23} \mathrm{~N}_{5} \cdot \mathrm{C}_{4} \mathrm{H}_{4} \mathrm{O}_{4}: \mathrm{C}, 58.60 ; \mathrm{H}, 6.99$; N, 17.98. Found: C, 58.06, H, 7.09; N, 17.72.

$N$-(7-Aminoheptyl)- $N$ ',-cyano- $N$ '-phenethylguanidine (5l): Yield $60 \%$; mp fumarate: $165-166{ }^{\circ} \mathrm{C}$; ${ }^{1} \mathrm{H}$ NMR $\left(\mathrm{D}_{2} \mathrm{O}\right) \delta:$ 1.13-1.20 (m, 8H), 1.45 (m, 2H), 2.67-2.85 (m, 6H), $3.28(\mathrm{t}, 2 \mathrm{H}), 6.49$ (s, 2H), 7.087.19 (m, 5H); EI-MS: $301.2(\mathrm{M})^{+}$; Anal. Calcd. for fumarate $\mathrm{C}_{17} \mathrm{H}_{27} \mathrm{~N}_{5} \cdot \mathrm{C}_{4} \mathrm{H}_{4} \mathrm{O}_{4} ; \mathrm{C}, 60.41 ; \mathrm{H}, 7.48 ; \mathrm{N}$, 16.77; Found: C, 60.16; H, 7.61; N, 16.76.

\section{Analgesic activity}

Male and Female (1:1) Kunming mice (weighing 18-22 g) were used to test the analgesic activity. The tests were carried out by administering the compounds orally at a dose of $40 \mathrm{mg} / \mathrm{kg}$, and each compound was tested on 10 mice. Thirty minutes later, the animals were injected intraperitoneally (ip) with $0.4 \mathrm{~mL} /$ mouse of a $0.6 \%$ acetic acid solution and writhes were counted during the following 15 min. The mean number of writhes for each experimental group and percent decrease compared with control group (10 mice treated with saline) were calculated.

\section{References}

1. Li, G.; Regunathan, S.; Barrow, C. J.; Eshraghi, J.; Copper, R.; Reis, D.J. Agmatine: an endogenous clonidine-displacing substance in the brain. Science 1994, 263, 966-969.

2. Raasch, W.; Regunathan, S.; Li, G.; Reis, D. J. Agmatine, the bacterial amine, is widely distributed in mammalian tissues. Life Sci. 1995, 56, 2319-2330.

3. Kolesnikov, Y.; Jain, S.; Pasternak, G. W. Modulation of opioid analgesia by agmatine. Eur. J. Pharmacol. 1996, 296, 17-22.

4. Aricioglu-Kartal, F.; Uzbay, I. T. Inhibitory effect of agmatine on naloxone-precipitated abstinence syndrome in morphine-dependent rats. Life Sci. 1997, 61, 1775-1781.

5. Li, J.; Li, X.; Pei, G.; Qin, B. Y. Agmatine inhibited tolerance to and dependence on morphine in guinea pig ileum in vitro. Acta. Pharmacol. Sin. 1998, 19, 564-568. 
6. Li, J.; Li, X.; Pei, G.; Qin, B. Y. Effects of agmatine on tolerance to and substance dependence on morphine in mice. Acta. Pharmacol. Sin. 1999, 20, 232-238.

7. Li, J.; Li, X.; Pei, G.; Qin, B. Y. Analgesic effect of agmatine and its enhancement on morphine analgesia in mice and rats. Acta. Pharmacol. Sin. 1999, 20, 81-85.

8. Su, R. B.; Li, J.; Qin, B. Y. A biphasic opioid function modulator: agmatine. Acta. Pharmacol. Sin. 2003, 24, 631-636.

9. Piletz, J. E.; May, P. J.; Wang, G.; Zhu, H. Agmatine Crosses the Blood-Brain Barrier. Ann. N.Y. Acad. Sci. 2003, 1009, 64-74.

10. John, C. R.; Brent, M. G.; Kelley, F. K.; Carolyn, A. F. Pharmacodynamic and Pharmacokinetic Studies of Agmatine after Spinal Administration in the Mouse. J .Pharmacol. Exp. Ther. 2005, 314, 1226-1233.

11. Perioli, L.; Ambrogi, V.; Bernardini, C.; Grandolini, G.; Ricci, M.; Giovagnoli, S.; Rossi, C. Potential prodrugs of non-steroidal anti-inflammatory agents for targeted drug delivery to the CNS. Eur. J. Med. Chem. 2004, 39, 715-727.

12. Masereel, B.; Wouters, J.; Pochet, L.; Lambert, D. Design, Synthesis, and Anticonvulsant Activity of 1-(Pyrid-3-ylsulfonamido)-2-nitroethylenes. J. Med. Chem. 1998, 41, 3239-3244.

13. Troschutz, R.; Karger, A. Synthesis of 6-Phenyl-Atevirdine. J. Heterocycl. Chem. 1997, 34, 11471151.

14. Schou, C.; Ottosen, E. R.; Petersen, H. J.; Bjorkling, F.; Latini, S.; Hjarnaa, P. V.; Bramm, E.; Binderup, L. Novel cyanoguanidines with potent oral antitumour activity. Bioorg. Med. Chem. Lett. 1997, 7, 3095-3100.

15. Perez-Medrano, A.; Buckner, S. A.; Coghlan, M. J.; Gregg, R. J.; Gopalakrishnan, M.; Kort, M. E.; Lynch, J. K.; Scott,V. E.; Sullivan, J. P.; Whiteaker, K. L.; Carroll, W. A. Design and synthesis of novel cyanoguanidine ATP-sensitive potassium channel openers for the treatment of overactive bladder. Bioorg. Med. Chem. Lett. 2004, 14, 397-400.

16. Falco, J. L.; Lloveras, M.; Buira, I.; Teixido, J.; Borrell, J. I.; Mendez, E.; Terencio, J.; Palomer, A.; Guglietta, A. Design, synthesis and biological activity of acyl substituted 3-amino-5-methyl1,4,5,7-tetrahydropyrazolo[3,4-b]pyridin-6-ones as potential hypnotic drugs. Eur. J. Med. Chem. 2005, 40, 1179-1187.

17. Cruciani, G.; Pastor, M.; Guba, W. VolSurf: a new tool for the pharmacokinetic optimization of lead compounds. Eur. J. Pharm. Sci. 2000, Suppl 2: S29-39.

18. Ooms, F.; Weber, P.; Carrupt, P. A.; Testa, B. A simple model to predict blood-brain barrier permeation from 3D molecular fields. Biochim. Biophys. Acta 2002, 1587, 118-125.

19. Rogelio P. F.; Gregory P. R. Synthesis and characterization of a novel ranitidine dimmer. Bioorg. Med. Chem. Lett. 1997, 7, 3045-3048.

20. Minamida, I.; Iwanaga, K.; Okauchi,T. EP302389, (1989).

21. Clitherow, J. W.; Price, B. J.; Bradsaw, J.; Martin-Smith, M. DE 2946332, (1980).

22. Minamida, I.; Iwanaga, K.; Tabuchi, T.; Uneme, H.; Danstuji, H.; Okauchi,T. Synthesis and insecticidal activity of acyclic nitroethene compounds containing a 3-pyridylmethylamino group. J. Pestic. Sci. 1993, 18, 31-40.

23. Southan, G. J.; Szabo, C.; Thiemermann, C. Isothioureas: potent inhibitors of nitric oxide synthases with variable isoform selectivity. Br. J. Pharmacol. 1995, 114, 510-516. 
24. Jozesf, R.; Laszlo, P. Synthesis of 1 - and 2-R $-3-\mathrm{R}^{2}, \mathrm{R}^{3}$-Amino-5-amino-1,2,4-triazoles. J. Heterocycl. Chem. 1986, 23, 401-408.

25. Zhang, H.B.; Huang, H. Y.; Huang, W. L.; Wang, Q. J. Synthesis and antidiabetic activity of sulfonylcyanoguanidines. Zhongguo Yaoke Daxue Xuebao 1999, 30, 243-245.

26. Sashadri, S.; Sanghavi, N. M.; Naik, R. V.; Tawate, S. R.; Trivedi, M. N.; Fruitwala, M. A. Syntheis and bactericidal activity of some symmetrical bisthioalkyl derivatives. Indian. J. Chem., Sect. B 1993, 32B, 688-692.

27. Magatti, C. V.; Doll, R. J. US 4803206 (1989).

Sample availability: Available from the authors.

(c) 2006 by MDPI (http://www.mdpi.org). Reproduction is permitted for noncommercial purposes. 